\title{
Impact of COVID-19 pandemic on maternity services and challenges faced by pregnant women delivering at a tertiary care centre in South India
}

\section{Parvathi Teja Naik ${ }^{1}$, Rupesh Bala Murugan ${ }^{1}$, Haritha Sagili ${ }^{1}$, Subitha Lakshminarayanan ${ }^{2}$, Priyadarshini Muruganandhan ${ }^{1}$, Aishwarya Thalappan Puliyullaveetti', Sathish Rajaa ${ }^{2}$, Sujithra Devi Radhakrishnan ${ }^{1 *}$}

${ }^{1}$ Department of Obstetrics and Gynecology, ${ }^{2}$ Department of Preventive and Social Medicine, JIPMER, Pondicherry, India

Received: 15 September 2021

Accepted: 30 September 2021

*Correspondence:

Dr. Sujithra Devi Radhakrishnan,

E-mail: sujiradjan@gmail.com

Copyright: (c) the author(s), publisher and licensee Medip Academy. This is an open-access article distributed under the terms of the Creative Commons Attribution Non-Commercial License, which permits unrestricted non-commercial use, distribution, and reproduction in any medium, provided the original work is properly cited.

\begin{abstract}
Background: Coronavirus disease 2019 (COVID-19), was declared a pandemic by the World Health Organization (WHO) on March 11, 2020. India entered a nationwide lockdown on March 25, 2020, disrupting regular health services. Hence this study was conducted to estimate the impact of the COVID-19 pandemic on maternity services and to describe the challenges faced by pregnant women.

Methods: A descriptive cross-sectional study conducted from July 2020 to October 2020 at tertiary care hospital, Puducherry, India. 422 pregnant women delivered during this period were included and detailed questionnaire was administered regarding antenatal, intrapartum, postnatal services and, challenges faced during the pandemic via telephonic call. Responses were documented in Epicollect version 5, transferred to Microsoft excel and analysed using Stata version 14.2. Continuous variables were expressed as mean (SD), and Categorical variables as proportions.

Results: The $100 \%$ response rate was achieved with $32.5 \%$ high risk pregnancies. The $71.8 \%$ women availed direct emergency medical services while $28.2 \%$. were referrals. The $79.9 \%$ and $25 \%$ of the women in third trimester had less antenatal visits and discontinued haematinics respectively. The $47.4 \%$ had first trimester testing, $30 \%$ didn't receive lactation support. $81 \%$ and $69 \%$ of primiparous and multiparous women respectively had adopted postpartum Intrauterine contraceptive device as contraception. The $35-50 \%$ women faced challenges in availing essential obstetric care services.

Conclusions: Development of robust linkage system between existing primary healthcare and tertiary care for managing both low and high-risk pregnancies is of paramount importance to alleviate maternal and perinatal mortality and morbidity.
\end{abstract}

Keywords: COVID-19, Challenges faced, Maternity services

\section{INTRODUCTION}

Coronavirus disease 2019 (COVID-19), caused by the severe acute respiratory syndrome coronavirus 2 (SARSCoV-2), was declared a pandemic by the WHO on March $11,2020 .{ }^{1}$ India entered a nationwide lockdown on March 25, 2020, as a measure for containment of COVID-19 infection. Globally the pandemic has partially or entirely disrupted the existing health services. In low-and middle-income countries (LMICs) like India, the impact of lockdown, quarantine, and diversion of the resources have disrupted essential health services, including maternal and child health care. ${ }^{2}$ The most crucial part of tackling this pandemic would 
be prioritising context-relevant essential health care for the vulnerable population, including pregnant women. The state of pregnancy usually involves immunological transformation and increased susceptibility to viral infections; therefore, there is a need to provide utmost care and support to pregnant women without exposing them to risk of SARS-CoV2. ${ }^{3}$ Antenatal-care services involve medical and surgical care for pregnant women, focusing on delivery and the immediate post-partum period and requires cooperation at various levels within the healthsystem to reduce adverse maternal and foetal outcome.

Several international and national organisations have released guidelines for antenatal, intrapartum and postpartum services during the pandemic. ${ }^{4-7}$ A modelling study estimated additional 12,200 maternal deaths in LMICs over 6 months following reduction of coverage of essential maternal health services by $9.8-18.5 \%{ }^{8}$ There are limited studies assessing the direct or indirect effect of COVID-19 on the care provided to pregnant women in India. A prospective study conducted in Western India demonstrated that COVID-19 has indirect effects on maternal and child health. ${ }^{9}$ Therefore, there is a need to evaluate and review the maternity services and the challenges faced to access essential obstetric care. Hence, this study was carried out to estimate effect of pandemic on maternity care received and to describe challenges faced in availing these services among pregnant women delivering at a tertiary care centre in South India.

\section{METHODS}

This was a single institute descriptive cross-sectional study conducted between July 2020 to October 2020 in a tertiary care hospital in South India where 1200-1500 vaginal deliveries and 350-400 caesarean sections are being performed monthly, accounting for total deliveries of approximately 1600-1900 per month. However, during the COVID-19 pandemic, all routine services were closed down for 8 months. Our institutioninitiated telemedicine services for continued maternity services and follow-up of high-risk pregnancies. Study was conducted after obtaining institute scientific advisory committee (JSAC/86/2020/484) and ethics committee approval (JIP/IEC/2020/273) and it confirms to the provisions of the declaration of Helsinki. Pregnant women aged 18 years and above who had delivered at our hospital were included in the study. The contact details of patients who had delivered from July 2020 to October 2020 were obtained from confinement registers. Approximately 20 deliveries were being conducted daily, and about five women were chosen by simple random sampling from the daily list. Random numbers were generated using Microsoft excel.

Due to a relative lack of estimates, sample size calculation was based on the assumption that $50 \%$ of women had received essential obstetric and contraceptive services during the COVID pandemic phase. With absolute precision of $5 \%$, a confidence level of $95 \%$ frequency in a population, using Openepi software version 3.0, the initial estimate was 384. After a corrected estimate (considering non-response of 10\%), the final sample size was 422 .

Women were contacted via telephonic call by the principal/co-investigator. Verbal telephonic consent was recorded, following which the investigator administered the questionnaire to collect the data inputs. Questionnaire was designed to capture services in 3 domains: antenatal, intrapartum, postnatal care services and challenges faced in availing these services. The questionnaire was piloted, and modifications were done accordingly. In addition, age, parity, place, socioeconomic status, occupation, COVID status of self/family member during the antenatal/delivery period, and clinical details were noted. Responses were entered in Epicollect ver 5. Data was then transferred from Epicollect 5 to MS excel and analysed using Stata version 14.2 (StataCorp, college park, TX, USA). Continuous variables expressed as mean (SD), and categorical variables expressed as proportions.

\section{RESULTS}

We contacted a total of 422 participants, and the response rate was $100 \%$. The socio-demographic and clinical characteristics of the pregnant women who had delivered in our hospital is described in Table 1.

Three hundred and three $(71.8 \%)$ of the pregnant women who had delivered from July 2020 to October 2020 had directly reported to our emergency medical services, and referrals from other centres were $28.2 \%$. The majority of them $(93.6 \%)$ reached our centre by private transport at their own expense, and $27(6.4 \%)$ had availed the government ambulance (108) services.

Table 2 depicts the essential obstetric care services availed by the pregnant women in our study. Two hundred eightyeight $(68.2 \%)$ women had confirmed their pregnancy by $6-$ 8 weeks of gestation; majority by a urine pregnancy test. $91.7 \%$ of them had their first contact/booking appointment with health care worker before 12 weeks of gestation for antenatal registration and only $35.5 \%$ had received antenatal counselling. Only $20.1 \%$ of pregnant women had in-person antenatal contact with HCWs in third trimester.

Less than half of pregnant women had undergone testing for haemoglobin, blood group, and urine examination in the first trimester. Twenty-one participants had ultrasonography in both first and second trimester, none of them had sonographic evaluation in third trimester. Almost all women were started on haematinics in second trimester, but only $2 / 3^{\text {rd }}$ continued taking them in the third trimester. All had reported to emergency services with labour pains, and were screened for COVID-19. Fourteen women $(3.3 \%)$ tested positive, were isolated in COVID19 designated ward for continued care and had an uncomplicated hospital stay. One hundred twenty-five women who underwent caesarean section failed to initiate 
breast feeding within 30 min either because of shifting of neonate to intensive care unit or themselves recovering from anaesthesia. The $81 \%$ and $69 \%$ of primiparous and multiparous women respectively had adopted a contraceptive method accounting for 3/4th of total participants $(n=327,77.4 \%)$. IUCD was used in $1 / 3$ rd of both primiparous multiparous women. $16.5 \%$ of multipara underwent tubectomy along with c-section.
The $35-50 \%$ women faced challenges in availing the essential obstetric care services (Table 3). On enquiring most of them had problems due to lockdown, containment zones, closure of nearest health facilities, lack of public transportation with a burden of additional expenditure and fixing teleconsultation appointments. These factors posed difficulties in access to health care worker/ investigations and procuring medicines.

Table 1: Socio-demographic and clinical characteristics of study population, $(n=422)$.

\begin{tabular}{|c|c|c|}
\hline Variables & $\mathbf{N}$ & Percentage $(\%)$ \\
\hline \multicolumn{3}{|l|}{ Age (years) } \\
\hline $18-35$ & 409 & 96.9 \\
\hline$\geq 35$ & 13 & 3.1 \\
\hline \multicolumn{3}{|l|}{ Education } \\
\hline No formal education & 1 & 0.2 \\
\hline Primary & 9 & 2.2 \\
\hline High school & 85 & 20.2 \\
\hline Higher secondary & 109 & 25.8 \\
\hline Graduate & 218 & 51.6 \\
\hline \multicolumn{3}{|l|}{ Socio-economic class ${ }^{a}$} \\
\hline Upper/upper middle & 14 & 3.3 \\
\hline Middle & 174 & 41.3 \\
\hline Lower/upper lower & 234 & 55.4 \\
\hline \multicolumn{3}{|c|}{ Nearest health center (min) } \\
\hline$<30$ & 412 & 97.6 \\
\hline$\geq 30$ & 10 & 2.4 \\
\hline \multicolumn{3}{|c|}{ Distance from our center $(\mathbf{k m})$} \\
\hline$<50$ & 290 & 68.7 \\
\hline $50-100$ & 71 & 16.8 \\
\hline$>100$ & 61 & 14.5 \\
\hline \multicolumn{3}{|l|}{ Obstetric index } \\
\hline Primigravida & 301 & 71.3 \\
\hline Multigravida & 121 & 28.7 \\
\hline \multicolumn{3}{|l|}{ Risk categorization } \\
\hline Low & 285 & 67.5 \\
\hline High & 137 & 32.5 \\
\hline
\end{tabular}

${ }^{\text {a }}$ Modified B. G. Prasad classification 2020

Table 2: Essential obstetrics care.

\begin{tabular}{|llll|}
\hline Variables & $1^{\text {st }}$ trimester & $2^{\text {nd }}$ trimester & $3^{\text {rd }}$ trimester \\
\hline Ante-partum & & & $<2 / \geq 2$ \\
\hline No. of antenatal visits & $0-1 />1$ & $36(8.5) / 386(91.5)$ & $337(79.9) / 85(20.1)$ \\
\hline Examination by HCW (including BP) & $24(5.7) / 398(94.3)$ & $412(97.6)$ & $420(99.5)$ \\
\hline Investigations & $397(94.0)$ & & $421(99.8)$ \\
\hline Blood: Haemoglobin and blood group & $200(47.4)$ & - & - \\
\hline GTT & - & $401(95.0)$ & - \\
\hline Urine: pregnancy test & $392(92.9)$ & - & - \\
\hline Microscopy/culture & $195(46.2)$ & - & - \\
\hline Ultrasonography & $21(5)$ & $393(93.1)$ & $306(75.5)$ \\
\hline Supplements & & & - \\
\hline Folic acid & $308(72.98)$ & - & - \\
\hline Iron/calcium & - & $418(99.0)$ & $301(71.3)$ \\
\hline Deworming & - & $401(95.0)$ & \\
\hline Tetanus toxoid vaccination & - & & \\
\hline
\end{tabular}

Continued. 


\begin{tabular}{|c|c|}
\hline Variables & $2^{\text {nd }}$ trimester \\
\hline Intra-partum & $\mathbf{N}(\%)$ \\
\hline Induction of labour & $123(29.2)$ \\
\hline Mode of delivery & SVD*-299 (70.8)/OVD**-6 (1.5)/CS***-117 (27.7) \\
\hline \multicolumn{2}{|l|}{ Post-partum } \\
\hline NICU admission & $88(20.8)$ \\
\hline Lactation support & $302(71.6)$ \\
\hline Breastfeeding within 30 minutes & $297(70.4)$ \\
\hline Iron and calcium supplements & $381(90.3)$ \\
\hline
\end{tabular}

Table 3: Challenges faced in accessing essential obstetric services.

\begin{tabular}{|lll|}
\hline Challenges & N & Percentage $(\%)$ \\
\hline Access to health care workers & 175 & 41.5 \\
\hline Closure of nearest health facility & 146 & 34.6 \\
\hline Procuring medicines & 150 & 35.5 \\
\hline Investigations & 149 & 35.3 \\
\hline Fixing teleconsultation appointment & 213 & 50.5 \\
\hline Travel to health facility & 145 & 34.4 \\
\hline
\end{tabular}

\section{DISCUSSION}

During COVID-19 pandemic first wave, there was significant fall in the total deliveries during the study period to one-third in comparison to pre-COVID delivery rate in our institution with closure of out-patient services and travel restrictions. Our study revealed several indirect effects on maternity services. Majority of the women could not have recommended no. of in-person visits to health care facility especially in their third trimester. In spite of high anaemia prevalence in our country, onefourth of the pregnant women had discontinued their haematinics supplements in third trimester. Also, postpartum we observed suboptimal lactation support and contraceptive counselling due to diversion of human resources to combat pandemic. Approximately half of the women faced challenges in availing essential obstetric care services including access to health care worker/laboratory and pharmacy facilities, closure of nearest health facilities, lack of public transport with a burden of additional expenditure and difficulty with teleconsultation.

Our study attempted to find the impact of pandemic in a hospital-based setting with specific focus on maternity services availed and challenges encountered by pregnant women who had delivered at our hospital. Community based studies exploring the primary care and maternity service linkages would further explore the gaps and guide the policymakers for continued care during such pandemic.

SARS-CoV2 has necessitated enforcement of lockdowns causing severe hindrance to travel and gatherings for the well-being of people and the methods of delivering health services have drastically changed even though the needs of the pregnant women have remained the same. In January 2013, the government of India under reproductive, maternal, newborn, child and adolescent health $(\mathrm{RMNCH}+\mathrm{A})$ and $\mathrm{WHO}$ emphasize on antenatal care for a positive pregnancy and safe motherhood experience. ${ }^{10,11}$ Traditionally prenatal visits involve minimum eight contacts with HCWs and more frequent visits in high-risk pregnancies and this has helped to decrease perinatal and maternal mortality with improved screening, diagnosis and management of highrisk maternal factors during pregnancy. To meet the continued needs of pregnant women during and postpandemic, ministry of health and family welfare (MoHFW) of India released guidelines on $14^{\text {th }}$ April 2020 taking into consideration the health system approach at different levels as per the zonal categorization i.e., containment, buffer and outside these zones. $^{12}$ Explored these components and found majority of the pregnant women couldn't access their HCWs for their routine antenatal visits due to closure of nearest health facilities both public and private due to lockdown and lack of public transport.

Fakari et al observed that pandemic has aggravated the mental stress and anxiety among expecting mothers, which can indirectly cause an increase in high-risk pregnancies. ${ }^{13}$ Similar stressful experiences were shared by antenatal mothers in the international survey by Meaney et al due to lack of access to prenatal care and social support. ${ }^{14}$ These anxiety issues are not just limited to women but has been experienced among their partners, midwives, medical practitioners and midwifery students according to a survey conducted in Australia by Bradfield et al. ${ }^{15}$ In addition, available government ambulance services (108) were mostly being utilized for COVID-19 related activities and with lack of public transportation facilities; laboring women faced a huge task to reach a healthcare facility with many women delivering in ambulances. ${ }^{16}$ In our study women expressed the stress of additional challenges in procuring the hematinic which were freely 
supplied in the health care facility in pre-COVID times and added burden of expenditure incurred for medications and travel using private transport. One-third of the women could not get lactation support in their postnatal period further adding to their anxiety and stress. Ceulemans et al reported $43 \%$ of women experienced lack of medical counselling and support with regard to breastfeeding in primary care in Belgium. ${ }^{17}$

Restricted mobility during COVID19 lockdown has created a lacuna in essential maternity services. Prior to the pandemic antenatal care services included inperson visit by women to healthcare facilities. Alternate health delivery system such as telehealth, modified outreach and home visits were promoted to fill the gap; in an effort to avoid overcrowding and decrease cross infection. On $25^{\text {th }}$ March 2020, Indian medical council along with NITI Aayog released the telemedicine practice guidelines for continuous health to address the needs of special population including pregnant women. ${ }^{18}$ In line with the guidance we integrated obstetric services to the existing telemedicine services at our centre since April 2020 for continued care of high-risk pregnancies during the pandemic. Most of existing studies have explored the feasibility as well as applicability of telemedicine in LMICs but needs more focus on associated barriers and unique challenges. ${ }^{19,20}$ Half of our study participants expressed their challenges encountered with digital health system from network or internet connectivity, fixing appointment, procuring haematinics prescribed through e-prescription etc.

Strengths of the study is an attempt to find the impact of pandemic in a hospital-based setting with specific focus on maternity services availed and challenges encountered by pregnant women who had delivered at our hospital.

\section{Limitation}

The study is single institute study, retrospective design and telephonic based interview posing difficulty as most of the women could not spare enough time from their domestic responsibilities and some had recall issues regarding challenges faced. Community based studies exploring the primary care and maternity service linkages would further explore the gaps and guide the policymakers for continued care during such pandemic.

\section{CONCLUSION}

Development of robust linkage system between existing primary healthcare and tertiary care for managing both low and high-risk pregnancies is of paramount importance to alleviate maternal and perinatal mortality and morbidity. Systematic implementation and utilisation of telemedicine to bridge gaps in essential maternity service is imperative in LMICs. National policy makers must include action plans balancing maternal and newborn services along with pandemic management.

Funding: No funding sources

Conflict of interest: None declared

Ethical approval: The study was approved by the Institutional Ethics Committee

\section{REFERENCES}

1. WHO Director-General's opening remarks at the media briefing on COVID-19. 2020. Available at: https://www.who.int/director-general/speeches/ detail/who-director-general-s-opening-remarks-atthe-media-briefing-on-covid-19---11-march-2020. Accessed on 3 June 2021.

2. World Health Organization. Maintaining essential health services: operational guidance for the COVID19 context interim, guidance. 2020. Available at: https://www.who.int/publications/i/item/WHO-2019nCoV-essentialhealthservices-2020.1. Accessed May 25, 2021.

3. Liu H, Wang LL, Zhao SJ, Kwak-Kim J, Mor G, Liao AH. Why are pregnant women susceptible to COVID19? An immunological view point. J Reprod Immunol. 2020;139:103122.

4. Novel Coronavirus 2019 (COVID-19). ACOG; 2020. Available at: https://www.acog.org/en/Clinical/ Clinical-Guidance/Practice-Advisory/Articles/2020/ 03/Novel-Coronavirus-2019. Accessed May 25, 2021.

5. Royal College of Obstetricians Gynaecologists. Guidance for antenatal and postnatal services in the evolving coronavirus (COVID-19) pandemic. RCOG;2020. Available at: https://www. rcog.org.uk/en/guidelines-research-services/ guidelines/coronavirus-pregnancy. Accessed May 25, 2021.

6. CDC. Coronavirus Disease 2019 (COVID-19). Centers for Disease Control and Prevention. 2020. Available at: https://www.cdc.gov/coronavirus/2019ncov/hcp/inpatient-obstetric-healthcareguidance.html. Accessed on May 25, 2021.

7. Indian Council of Medical Research. Guidance for management of pregnant women in covid-19 pandemic. India: ICMR; 2020. Available at: https://www.icmr.gov.in/pdf/covid/techdocGuidance _for_management_of_pregnant_women_in_COVID 19_pandemic_12042020.pdf. Accessed May 25, 2021.

8. Roberton T, Carter ED, Chou VB. Early estimates of the indirect effects of the COVID-19 pandemic on maternal and child mortality in low-income and middle-income countries: a modelling study. Lancet Glob Health. 2020;8(7):e901-8.

9. Goyal M, Singh P, Singh K, Shekhar S, Agrawal $\mathrm{N}$, Misra S. The effect of the COVID-19 pandemic on maternal health due to delay in seeking health care: Experience from a tertiary center. Int J Gynaecol Obstet. 2021;152(2):231-5. 
10. $\mathrm{RMNCH}+\mathrm{A}$. A Strategic approach to reproductive, maternal, newborn, child and Adolescent health $(\mathrm{RMNCH}+\mathrm{A})$ in India. 2013. Available at: https://www.cghealth.nic.in/nhmcg/Informations/RM NCH/1_RMNCHA_Strategy.pdf. Accessed June $10,2021$.

11. WHO recommendations on antenatal care for a positive pregnancy experience. Geneva: World Health Organization.2016 Available at: https://apps.who. int/iris/bitstream/handle/10665/250800/WHO-RHR16.12-eng.pdf. Accessed June 10, 2021.

12. Guidance Note on Provision of Reproductive, Maternal, New born, Child, Adolescent Health Plus Nutrition (RMNCAH+N) services during and post COVID-19 Pandemic. Available at: https://www.mohfw.gov.in/pdf/GuidanceNoteonProv isionofessentialRMNCAHNServices24052020.pdf. Accessed June 22, 2021.

13. Rashidi Fakari F, Simbar M. Coronavirus pandemic and worries during pregnancy; a letter to Editor. Arch Acad Emerg Med. 2020;8(1):e21.

14. Meaney S, Leitao S, Olander EK, Pope J, MatvienkoSikar K. The impact of COVID-19 on pregnant womens' experiences and perceptions of antenatal maternity care, social support, and stressreduction strategies. Women Birth. 2021:S18715192(21)00079-2.

15. Bradfield Z, Wynter K, Hauck Y, Vasilevski $\mathrm{V}$, Kuliukas L, Wilson AN et al. Experiences of receiving and providing maternity care during the COVID-19 pandemic in Australia: A five-cohort cross-sectional comparison. PloS one. 2021;16(3):e0248488.
16. The Wire. COVID-19 Lockdown: Guidelines are not enough to ensure pregnant women receive care. Available at: https://thewire.in/women/covid-19lockdown-pregnantwomen-childbirth. Accessed June 20, 2020.

17. Ceulemans M, Verbakel JY, Van Calsteren K, Eerdekens A, Allegaert K, Foulon V. SARS-CoV2 Infections and Impact of the COVID-19 Pandemic in Pregnancy and Breastfeeding: Results from an Observational Study in Primary Care in Belgium. Int J environmental res public health. 2020;17(18):6766.

18. Board of Governors In supersession of the Medical Council of India Telemedicine Practice Guidelines Enabling Registered Medical Practitioners to Provide Healthcare Using Telemedicine. 2020. Available at: https://www.mohfw.gov.in/pdf/Telemedicine.pdf. Accessed June 23, 2021.

19. Zangmo R, Kumari A, Garg D, Sharma KA. Redesigning routine antenatal care in low resource setting during COVID-19 pandemic. Journal of family medicine and primary care. 2020;9(9):454751.

20. Garg S, Gangadharan N, Bhatnagar N, Singh MM, Raina SK, Galwankar S. Telemedicine: Embracing virtual care during COVID-19 pandemic. J family med primary care. 2020;9(9):4516-20.

Cite this article as: Naik PT, Murugan RB, Sagili H, Lakshminarayanan S, Muruganandhan P, Puliyullaveetti AT et al. Impact of COVID-19 pandemic on maternity services and challenges faced by pregnant women delivering at a tertiary care centre in South India. Int J Reprod Contracept Obstet Gynecol 2021;10:4090-5. 